\title{
Network Layer Mobility: an Architecture and Survey*
}

\author{
Pravin Bhagwat ${ }^{\dagger}$ Satish Tripathi ${ }^{\dagger}$ Charles Perkins ${ }^{\ddagger}$
}

CS-TR-3570

UMIACS-TR-95-117

September 13, 1995
${ }^{\dagger}$ Computer Science Department
University of Maryland
College Park, MD 20742

${ }^{\ddagger} \mathrm{IBM}$, T.J. Watson Research Center

\begin{abstract}
In this paper we explore various network layer concepts that pertain to the design of mobile networking systems. We show that mobility is essentially an address translation problem and is best resolved at the network layer. We have identified the fundamental services that must be supported at the network layer to carry out the task of address translation. Using these service primitives as building blocks, we propose a network layer architecture which enables smooth integration of mobile end systems within the existing Internet. The architecture is modularized into well-defined logical components. In this paper our objective is not to propose a specific scheme for supporting mobility, rather it is to highlight and analyze the essential aspects of supporting mobile end-systems, as well as to better understand the trade-off between various design alternatives.
\end{abstract}

${ }^{*}$ This work was supported in part by NSF grant CCR 9318933 


\section{Introduction}

Mobile end-systems frequently change their point of attachment to the network. In such an environment, in order for mobile devices to run without disruption, a universal networking infrastructure is needed. In addition, a common networking protocol is required which can support network-wide mobility. Mobile devices also need to communicate with the existing pool of information servers and file servers, which means that internetworking solutions for connecting stationary and mobile systems are also required. Unfortunately, the Internet Protocol (IP), which forms the fabric of the current world-wide data communication network, falls short of meeting this demand. The current Internet suite of protocols (TCP/IP) were designed under the assumption that end-systems are stationary. If during an active network session one end of the connection moves, the network session breaks. Naturally, all networking services layered on top of TCP/IP are also disrupted when end-systems become mobile. There are two approaches for solving this problem. One is to completely redesign internetworking protocols with the specific goal of supporting mobile end systems. The other approach is to provide additional services at the network layer in a backward compatible manner which make mobile internetworking possible. The first approach, though an interesting possibility from a research viewpoint, is infeasible since it would require radical changes to the currently deployed networking infrastructure. It is the latter approach that is the focus of our investigation.

To ensure inter-operability with the existing infrastructure, the handling of mobility should be completely transparent to the protocols and applications running on stationary hosts. In other words, from a stationary end-system's perspective, a mobile host should appear like any other stationary host connected to the Internet. This means the same naming and addressing conventions, those originally developed for stationary hosts, must apply to mobile hosts. In addition, any changes in a mobile's network attachment point should be completely hidden from the protocols and applications running on stationary hosts.

In this paper we explore various network layer concepts that pertain to the design of mobile networking systems. We show that mobility is essentially an address translation problem and is best resolved at the network layer. We have identified the fundamental services that must be supported at the network layer to carry out the task of address translation. Using these service primitives as building blocks, we propose a network layer architecture which enables smooth integration of mobile end systems within the existing Internet. The architecture is modularized into well-defined logical components. In this paper our objective is not to propose a specific scheme for supporting mobility, rather it is to highlight and analyze the essential aspects of supporting mobile end-systems, as well as to better understand the trade-off between various design alternatives.

\section{Internet Naming and Addressing}

The Internet is a large collection of networks which share the same address space and inter-operate using a common sets of protocols, such as TCP/IP [14, 15]. A fundamental concept of the Internet architecture is that each host ${ }^{1}$ has a unique network address, by which it is reachable from other hosts in the network. Data are carried in the form of packets which contain source and destination addresses. To communicate with another host, a source only need to know the address of the

\footnotetext{
${ }^{1}$ In the Internet jargon, host means an end-system connected to the Internet
} 
destination. It is the responsibility of the internet routing system to carry packets from a source to a destination node.

Internet routers maintain a view of network topology in the form of routing tables. These tables are consulted when making packet routing decisions. The process of routing involves inspecting the the destination address contained in the packet and, based on the contents of the routing table, determining the next-hop router to which packet should be relayed. Each router along the path from a source to a destination node repeats this process until the packet is finally delivered to the destination host.

If host addresses are treated as flat identifiers, routers will be required to maintain routing information on a per-host basis. Obviously, this is not feasible, given the large number of hosts (over 80 million!) that are connected to the Internet. A natural solution is to impose a hierarchy on the address structure. The purpose of hierarchical addressing scheme is to allow aggregation of routing information; higher layers in the hierarchy (e.g., routers) need only concern themselves with the portion of the address that is relevant at that layer. Hierarchical addressing is essential if the routing architecture is to be scalable. The Internet, for example, deploys a two-level hierarchical addressing scheme.

\subsection{Internet Addressing}

Each host in the Internet is assigned a unique 32-bit internet address (also known as an IP address) which consists of two parts: network-id and host-id. The boundary between the network-id and the rest of the address is a fixed location determined by the leading bits of an address (as shown in Figure 1). IP addresses are commonly represented using dotted notation where each octet is represented as a decimal number and dots are used as octet separators.

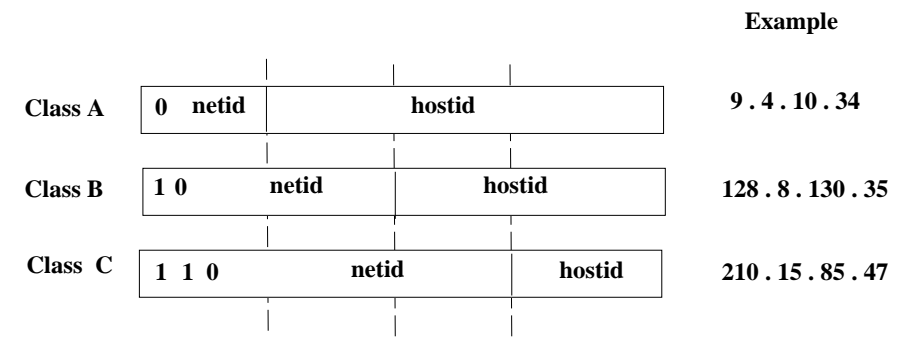

Figure 1: IP address structure

Under the current Internet addressing scheme, routers only need to maintain network topology information at the granularity of individual networks. This means only the network part of the destination address is used in making routing decision. Though hierarchical addressing makes routing simple and manageable, as a natural consequence, it puts certain restriction on the address usage. A hierarchical address can only be used within the domain of its definition. For example, an Internet address is only meaningful so long as the host using it remains connected to that network denoted by the network-id part of the address. When the host moves to a new network, it must be allocated a new address which is derived from the address space of the new network. In order for the Internet routing to work:

A mobile host must be allocated a new address when it moves. 


\section{$2.2 \quad$ Naming}

A related concept for identifying hosts in the network is Name. Names are user defined aliases (strings of characters) which are used to denote hosts. For example, ballast is the name of the file-server in our department, and its address is 128.8.128.88. An important distinction between names and addresses is that addresses are protocol specific (e.g., an IP address, CLNP address, IPX address, XNS address), but names are not. Names provide a way for applications to make reference to network entities without having to know anything about the underlying network protocol in use. This is useful, since users find names easier to use and remember than cumbersome network addresses.

Though applications refer to end systems by names, when packets are transported through the network they must contain addresses of destination nodes. This is because routers do not understand names, they can only interpret addresses. A translation mechanism, therefore, is required for mapping host names to addresses. To accommodate a large, rapidly expanding set of names, a decentralized naming mechanism called the Domain Name System (DNS) was deployed in the Internet. DNS stores name to address mappings in a distributed data structure. Finding the address of the host is essentially a directory lookup operation (see Figure 2). When two hosts on the Internet need to communicate with each other, the source node performs a DNS lookup to obtain the destination node's address and then initiates a connection setup procedure. During connection setup, each end of the connection learns about the address of the other end. So long as the connection is active, no additional DNS lookups are performed, since name to address binding is assumed to be static and is not expected to change during a connection lifetime.

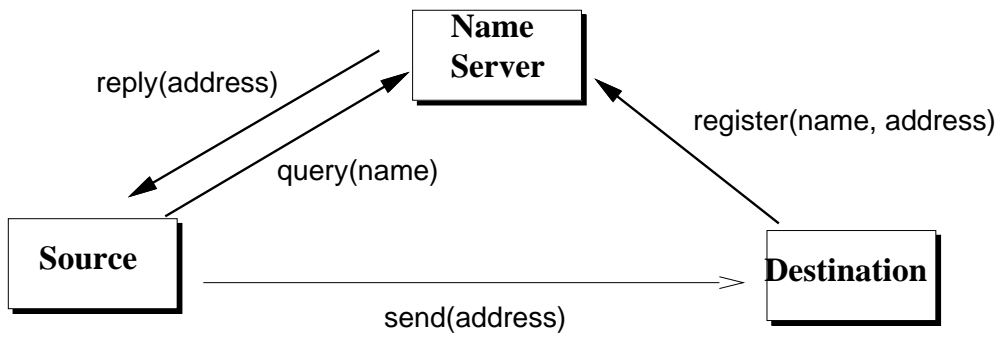

Figure 2: DNS based Name to Address resolution

\section{The Mobility Problem}

To illustrate why host mobility poses problem at the network later, it is important to emphasize the distinction between the concepts of name and address.

- Name: is a location independent identifier of a host. E.g. 'mimsy' is the name of the mailserver in our department.

- Address: indicates the location of a given host. E.g. mimsy's address 128.8.128.8 indicates that it is connected to network 128.8 .128

Names remain fixed regardless of where a host is located. An address on the other hand reflects a host's point of attachment to the network. For hosts that remain static throughout their lifetime, 
both names and addresses can be used interchangeably. For a mobile host, however, an address cannot be used as a unique identifier, since it must change with the location of the host. Name is the only location independent identification mechanism that can be used at the network layer to make references to mobile hosts.

\subsection{Mobility Problem: Directory Service View}

In networks where hosts are static, name to address bindings never change. Host mobility makes this binding a function of time. Therefore, network layer mechanisms are required for resolving names into addresses and tracking the location of hosts as they move. The Domain Name System (DNS), which provides name to address translation service in the Internet today, should be enhanced to meet the additional demands. However, this task is made difficult by many hurdles:

- The DNS has no provision to handle dynamic updates. This is because it was originally designed to provide name lookup service for stationary hosts only.

- The DNS design attempts to optimize the access cost, and not the update cost. Server replication and client caching provides significant performance gains for access only systems, but results in very poor performance when updates are performed. In a mobile environment, both updates and accesses are equally likely.

- DNS clients cache DNS records to reduce latency for future accesses and to reduce load on the name servers. There is no call back mechanism from servers to clients, in case cache entries become invalid.

A design for a distributed location directory service for mobile hosts was proposed by Awerbuch and Peleg in [2]. They formally proved an important theoretical result which established that a system cannot optimize both access and update operations ${ }^{2}$. Using the concept of Regional Directories (a type of cache) they proposed a distributed directory layout which guarantees that the communication overhead of access and update operations is within a poly-logarithmic factor of the lower bound.

As far as the Internet is concerned, distributed directory service based solutions do not appear very attractive since they cannot be deployed without changing existing host software. The Internet has already grown over 80 million hosts in population, which makes any change to host software almost impossible to achieve. Hence, an alternate solution method is required.

\subsection{Mobility Problem: Internet View}

When the Internet suite of protocols were originally developed, it was implicitly assumed that the name to address binding remained static. Thus, instead of referring to hosts through names, protocols were developed that referred to hosts through their addresses. A classic example is a TCP connection which is identified by a 4-tuple:

$<$ source IP address, source TCP port, destination IP address, destination TCP port >

\footnotetext{
${ }^{2}$ In their paper they use terms Find and Move to denote these operations.
} 
If neither host moves, all components of the connection identifier will remain fixed, and thus a continuous TCP session can be maintained between the two hosts. If either end of the connection moves, we run into the following problem:

- If the mobile host acquires a new IP address, then its associated TCP connection identifier also changes. This causes all TCP connections involving the mobile host to beak.

- If the mobile host retains its address, then the routing system cannot forward packets to its new locations.

The fundamental problem is that in the Internet architecture, an IP address serves dual purposes. From the transport and application layer perspective, it serves as an end-point identifier, and at the network layer, the same IP address is used as a routing directive. This problem is not specific to the Internet architecture; in fact all contemporary connection-less network architectures, such as OSI, IPX, and XNS, suffer from this problem. Since our objective is to ensure that connection do not break when hosts move, we can say that:

In order to retain transport layer sessions, a mobile host's address must be preserved regardless of its point of attachment to the network.

An immediate consequence of this choice is that we can not rely on the existing routing system for delivering packets to a mobile host's new location. A solution might be to keep per-mobile-host routing information at all routers, but this completely breaks the hierarchical model of routing, causing unbounded growth in the size of routing tables. Thus, the problem of supporting mobile hosts within the Internet is not just keeping track of hosts. In addition, it has to do with designing a mechanism for packet forwarding to mobile hosts without modifying and compromising the scalable nature of the Internet routing mechanism.

\section{Network Layer Solution Architecture}

In this section we describe a network layer architecture that allows smooth integration of mobile end-systems within the Internet. Our objective is to highlight and analyze the essential aspects of providing mobility extensions in any connection-less network; the specific details involved in designing a mobile-networking system will be discussed later. For ease of exposition, we will first introduce a few definitions.

Mobile Host: An internet host is called a Mobile Host $(M H)$ if it frequently changes its point of attachment to the network. A change in the attachment point can also happen while one or more transport layer sessions involving the $\mathrm{MH}$ are in progress. It is assumed that the rate of change of location is slower than the time it takes to for the mobile routing protocols to learn about the mobile host's new location.

Home Address: Like any other internet host, a mobile host is also assigned an internet address which is referred to as its Home Address (HA). A standard 32-bit internet address is allocated using the same guidelines that apply to stationary hosts. When the DNS is queried with a mobile host's name, it returns the home address of the mobile host. 


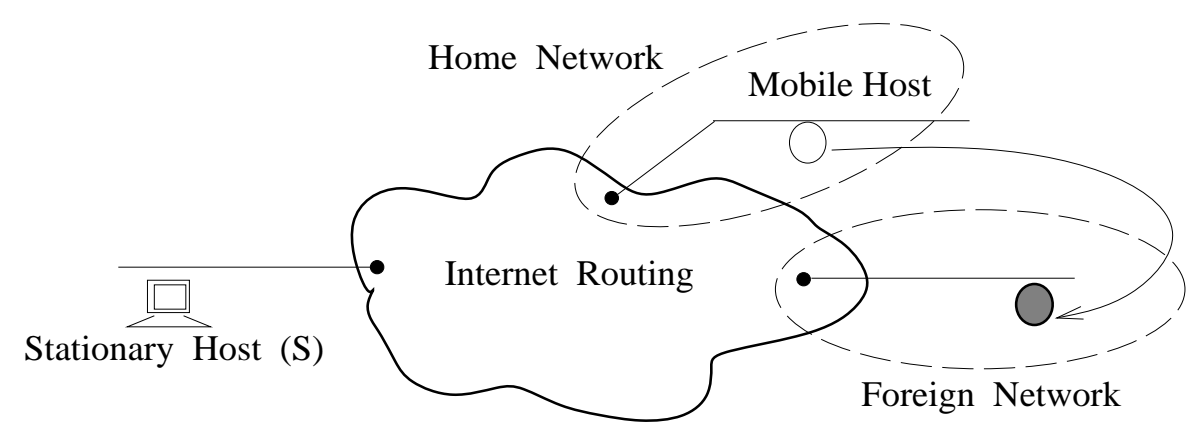

Figure 3: Tllustration of Terms

Home Network: Within each administrative domain, network administrators find it easier to reserve one or more subnetwork(s) for mobile hosts. The home address of a mobile host is allocated from the address space of one of these subnetworks, referred to as the Home Network in the subsequent discussion. The terms home address and home network also apply to stationary hosts. The only difference is that stationary hosts always remain connected to their home network, while mobile hosts sometimes may not be found at their respective home networks.

Foreign Network: Any connected segment of an Internet, other than the home network of a mobile host, to which the mobile host is allowed to attach is referred to as a Foreign Network. If $I$ denotes the set of all networks connected to the Internet, then any network in the set $I-$ $\{$ Home Network $\}$ is a foreign network to all hosts that derive their home addresses from the Home Network.

Notice that above definitions are relative to a mobile host. The same network could operate both as a home and as a foreign network, depending on which mobile host is connected to it. So long a mobile host remains connected to its home network, existing internet routing mechanism are sufficient to route packets up to its current location. It is only when it moves to a foreign network that additional mechanisms are required. If a mobile host moves within its home network (e.g., detach from one ethernet point and attach through another ethernet point), it does not constitute a move from the network layer point of view. Existing link layer bridging mechanism are capable of routing packets up to end-systems so long as they remains connected to the same layer 2 segment ${ }^{3}$.

In the previous section, we made two crucial observations:

1. The home address of a mobile host cannot be used for routing packets to its current location (except when it is attached to its home network).

2. A mobile host's address must be preserved in order to retain all active transport connections involving the mobile host.

\footnotetext{
${ }^{3} \mathrm{~A}$ collection of link layer networks, which are interconnected through bridges, is called a layer 2 segment. Within a layer 2 segment, a packet can be delivered solely on the basis of the destination node's link layer address; the network layer routing is not required
} 
These are two conflicting requirements. From the first observation, when a host moves, a new address, reflecting its new point of attachment to the network, must be used for the purpose of routing. The second observation says just the opposite: the original address must be preserved to retain all active network sessions.

\subsection{Two Tier Addressing}

We introduce the concept of two-tier addressing to resolve the problem associated with the dual use of an internet address. Our solution involves associating two internet addresses with each mobile host(see Figure 4). The first component of the address reflects the mobile's point of attachment to the network while the second component denotes its home address. The first address component serves as a routing directive. It changes whenever a mobile host moves to a new location. The second component of the address serves as an end-point identifier. It remains static throughout the lifetime of a mobile host. The purpose of two-tier addressing is to decouple the dual role of an internet address into two disjoint, well defined functions.

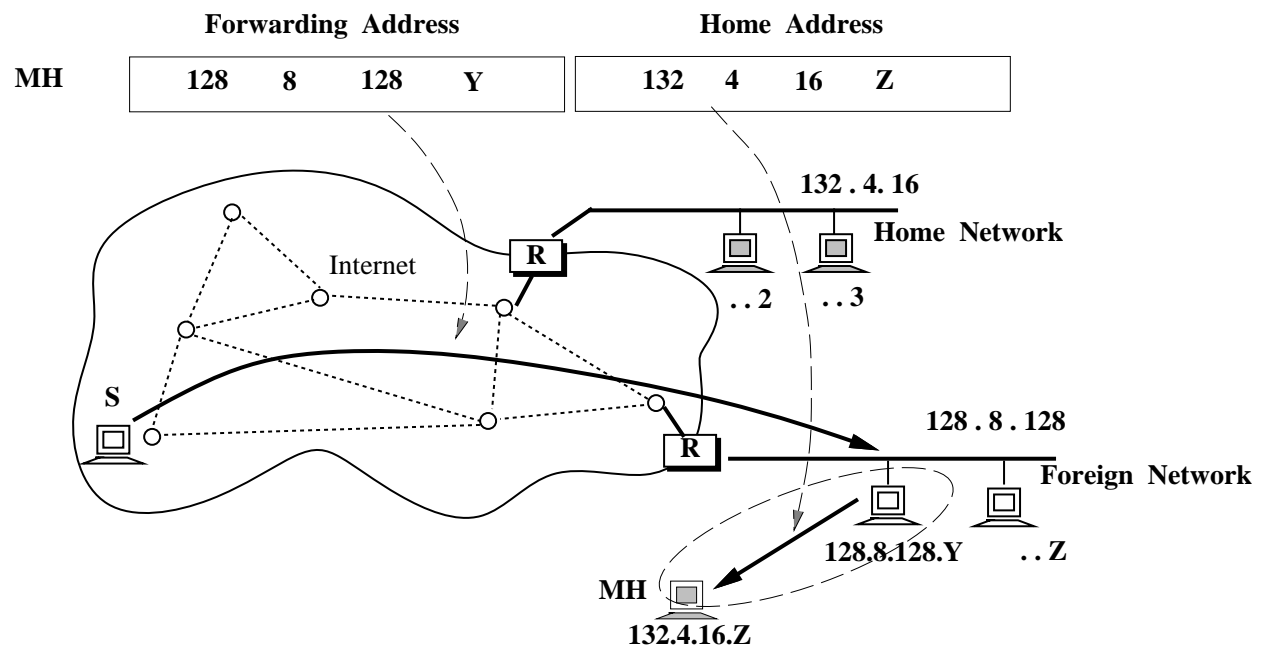

Figure 4: Two Tier addressing for Mobile Hosts

The concept of two-tier addressing is illustrated in Figure 4. Packets that are destined to mobile hosts contain the destination address in the two-tier format. The Internet routing system only looks at the first component of the address and routes those packets to the point where the mobile host is attached. At this point, the first address component is discarded. Only the second address component, the home address of the mobile host, is used in subsequent protocol processing. From an end-host's perspective this means that it notices no difference when it is attached to its home versus when it is located in a foreign network. In other words, the mobile host virtually remains connected to its home. Packets which originate from the mobile host and are destined to the stationary host $(S)$ do not require any special handling, since the Internet routing system can deliver those packets based on their destination addresses. If $S$ is also mobile, then the same two-tier addressing mechanism can be used to route packets to its current location.

It is important to note that the two-tier addressing is only a logical concept. Its realization doesn't necessarily require carrying two addresses in the destination address field of the network 
layer packets. In fact, doing so would require changes in the existing packet formats, necessitating changes to host and router software. It is desirable to support the two-tier addressing method using the existing mechanism available in the Internet Protocol suite. Below we describe how this can be achieved.

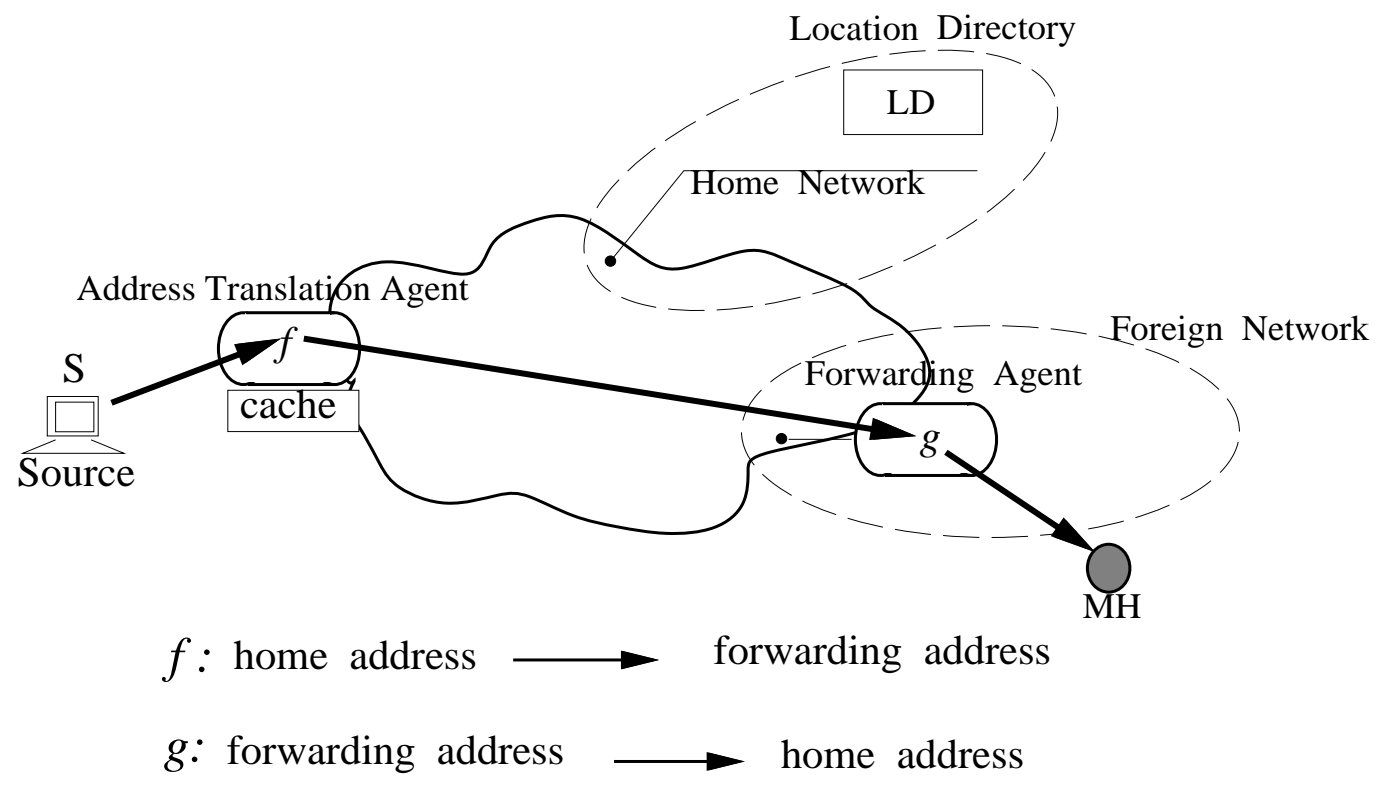

Figure 5: Packet Forwarding Model

\subsection{Architecture Components}

\subsubsection{Forwarding Agent (FA)}

When away from its home network, a mobile host can attach to the Internet through a foreign network. For the purpose of forwarding datagrams to its new location, an address derived from the address space of the foreign network must be used. Packets destined to the mobile host contain the address of a Forwarding Agent $(F A)$ in the forwarding address sub-field of the two-tier address. An FA provides an access point through which mobile hosts can attach to the network. It receives packets on behalf of mobile hosts, and forwards them to appropriate mobile hosts after necessary protocol processing.

Conceptually, the processing at the FA involves stripping the forwarding address part of the two-tier address and exposing the home address of the mobile host. Once the packet arrives at the FA, the forwarding address is no longer required in the subsequent protocol processing. When a packet arrives at the FA, it contains the address of the FA in its destination address field. The FA, essentially, maps the contents of the destination address (the forwarding address) to the home address of the associated mobile host. We use the notation $g$ to denote this mapping function:

$$
g:(\text { forwarding address }) \rightarrow(\text { home address })
$$

An FA should be able to relay packets to the mobile host on the basis of its home address. This is easy if the FA and the $\mathrm{MH}$ are directly connected (normally over a wireless link). Otherwise, the 
routing protocol operating in the foreign network should advertise host specific routing information within the foreign network to facilitate routing of these packets to mobile hosts. Normally, we would expect a wireless base station to operate as an FA in which case both the MH and the FA would be directly connected over a wireless link.

A mechanism is required so that mobile hosts can discover the identity of an FA when they connect to a foreign network. Similarly, a mechanism is required so that the FA can determine the identities of all mobile hosts that require its service. The simplest way to achieve this is through a route advertisement and a registration protocol. Forwarding agents periodically advertise their presence in the foreign network. Beaconing, the periodic broadcast of messages over the wireless medium, is the most commonly used method. Mobile hosts can listen to broadcasts, determine the identity (address) of the nearest FA, and initiate a registration sequence.

\subsubsection{Location Directory (LD)}

The component in the architecture that records the association between the home and the forwarding address of a mobile host is called a Location Directory (LD). The LD contains the most up-to-date mapping between a mobile host and its associated FA. Mobile Hosts are required to send updates to the LD whenever they moves to a new location.

Since the number of mobile hosts is expected to be very large, a centralized realization of the LD is deemed infeasible. A policy for distributing LD components should take many factors into consideration, such as the cost of access, ease of locating LD components, and security and ownership of location information. Since the LD will be accessed very frequently, a good distribution method should exploit the locality of access patterns and provide uniform load balancing among all LD components. Given a model for the LD access pattern, the LD distribution can be formulated as an optimization problem[1]. Unfortunately, these mathematical results $[1,4,3]$ cannot be directly applied in the Internet. The primary reason is that in the Internet factors such as ease of location, security, and ownership take precedence over any cost optimization considerations.

A feasible distribution scheme in the Internet is the owner-maintains-rule. According to this scheme, the LD entries for mobile hosts are maintained at their respective home networks. Within each home network, a good place for locating an LD component is at the home router. Advantages of this scheme are:

1. Each home network is responsible for maintaining, securing, authenticating, and distributing LD information for its mobile hosts. This policy fits well within the Internet philosophy of autonomous operation.

2. No special mechanisms are required to locate the LD components. It is important to point out that in a distributed scheme, in order for a source to send a query to the right LD component, the source is required to know the address of the LD component in advance. Under the ownermaintains-rule, a source simply sends a query that is addressed to the mobile host. The packet is delivered to the home network by normal internet routing where it is intercepted by the home router and subsequently relayed to the correct LD component.

This is certainly not the only possible distribution scheme. Later in this paper we'll discuss other options while reviewing various MobileIP proposals. 


\subsubsection{Address Translation Agent (ATA)}

Hosts that need to communicate with a mobile host insert the mobile's home address in the destination address field of all packets they issue. At some point during the routing process this address should be replaced by the address of the FA associated with the mobile host. The entity which performs this operation is called an Address Translation Agent. The process of address translation involves querying the LD, obtaining the FA address, and subsequently making use of this address in forwarding packets to the correct location of the mobile host. The address translation function is:

$$
f:(\text { home address }) \rightarrow(\text { forwarding address })
$$

From a two-tier addressing perspective, an ATA initializes the forwarding address part of the destination address. In an actual implementation this could be achieved by replacing the original destination address of the packet with the FA's address. This operation can be performed at the source host; however, the only problem is that the function $f$ cannot be computed without making changes to the existing host software of millions of hosts.

For performance reasons, an ATA may decide to cache LD entries which are frequently used in making forwarding decisions. Querying the LD before making each address translation operation could be prohibitively expensive, particularly so when the ATA and the LD are geographically separated. Caching, however, introduces a new requirement in the architecture; that of maintaining consistency between the LD and its cached entries throughout the Internet.

\subsection{Location Update Protocol (LUP)}

Keeping the LD up-to-date in the face of frequently changing host location is crucial. Keeping cached LD entries consistent with the master LD is an equally important consideration. Inconsistencies could make mobile hosts inaccessible and even cause the formation of routing loops. The purpose of Location Update Protocol(LUP) is to provide reliable mechanisms for keeping the LD and its cached copies consistent at all times.

To a large extent, the choice of the LUP depends on the caching policy used. Together, they determine the scalability and routing characteristics of a mobility solution. In systems which do not permit LD caching, ATAs must be co-located with the LD, since issuing an LD query for each packet that an ATA forwards is prohibitively expensive. In such systems, packets addressed to mobile hosts first travel all the way up to the home network before any address translation (operation $f$ ) is performed. Clearly, the paths that packets follow are non-optimal in this case. Caching improves the routing efficiency of a mobile networking system, as packets do not have to travel to home networks before being forwarded toward the FAs associated with the destinations. At the same time, caching makes the system more complex and vulnerable to security attacks. If cache entries are not properly authenticated, it is possible to redirect packets away from a mobile host and cause denial-of-service.

\subsection{Packet Forwarding Operation}

With the inclusion of address translation agents and forwarding agents, the operation of packet forwarding can be easily illustrated. Figure 5 illustrates how packets from a stationary host $(S)$ are routed to a mobile host $(M H)$. $S$ sends out packets which are addressed to the home address of the $M H$. These are intercepted by an address translation agent which maps (using function $f$ ) 
the original destination of the packet to the address of the forwarding agent. Once these packets arrive at the forwarding agent, the FA remaps (using function $g$ ) the destination to the home address of the mobile host and delivers them to the mobile host. Along the path from the source to the destination, packets twice undergo an address translation operation. The end result of this translation process, the function gof, is an identity mapping, which means that the whole process of address translation is completely transparent to hosts located at both ends of the path. They communicate as if they were stationary. The transport layer protocols and the applications running on stationary as well as mobile hosts operate without any modifications whatsoever. This property of the solution architecture is termed as transport layer transparency.

The proposed architecture preserves transport layer transparency regardless of where and how in the network the LD, ATAs, and FAs are distributed. This flexibility enables us to capture the design choices made in other MobileIP proposals. Later in this paper, we'll show that each one of these proposals can be viewed as a special case of the proposed architecture.

\subsection{Address Translation Mechanisms}

So far we described how various components of the architecture co-operate amongst each other to perform necessary address translation operations. The actual mechanisms for effecting those were not mentioned. Within the Internet architecture there are two possible ways of doing it: either using encapsulation or using loose source routing. A brief description of both follows:

\subsubsection{Encapsulation}

In the encapsulation method a new packet header is appended at the beginning of the original packet (see Figure 6). The outer header contains the address of the forwarding agent while the inner header contains the home address of the mobile host. Since the Internet routing system only looks at the outer packet header, it routes this packet to the forwarding agent. The forwarding agent strips the outer packet header and delivers the inner packet locally to the mobile host.

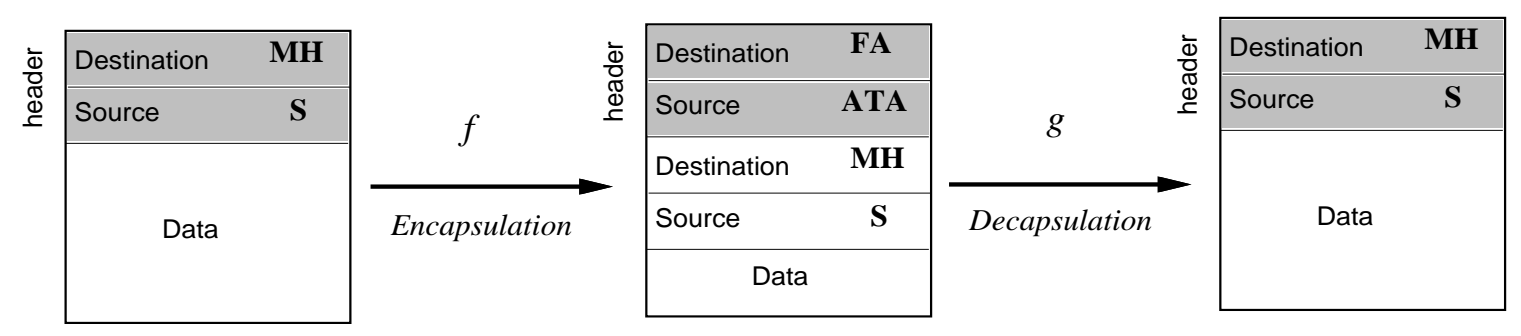

Figure 6: Illustration of Encapsulation and Decapsulation

\subsubsection{Loose Source Routing (LSR)}

Loose Source Routing is an option that is supported in IP which can also be used to perform address translation operation ${ }^{4}$. Using IP's source routing option, an address translation agent can cause packets addressed to a mobile host's home address to be routed via a forwarding agent. Figure 7

\footnotetext{
${ }^{4}$ Originally it was included in IP not for this purpose, but to help in debugging network problems
} 
illustrates how this is done. An LSR option is used to specify a nil terminated list of addresses. The Internet routing system routes the packet containing the LSR option to each address, one by one, in the sequence it appears in the list. The current destination is kept in the destination address field of the packet header and a pointer points to the address which is to be visited next in the sequence. When the packet arrives at the current destination, the contents of the destination address field are swapped with the address pointed by the next hop pointer, and, the pointer is advanced to the next address in the list. This process is repeated until the packet is delivered to the address which occurred last in the original list of addresses included in the LSR option. At this point the the next hop pointer in the LSR option points to nil.

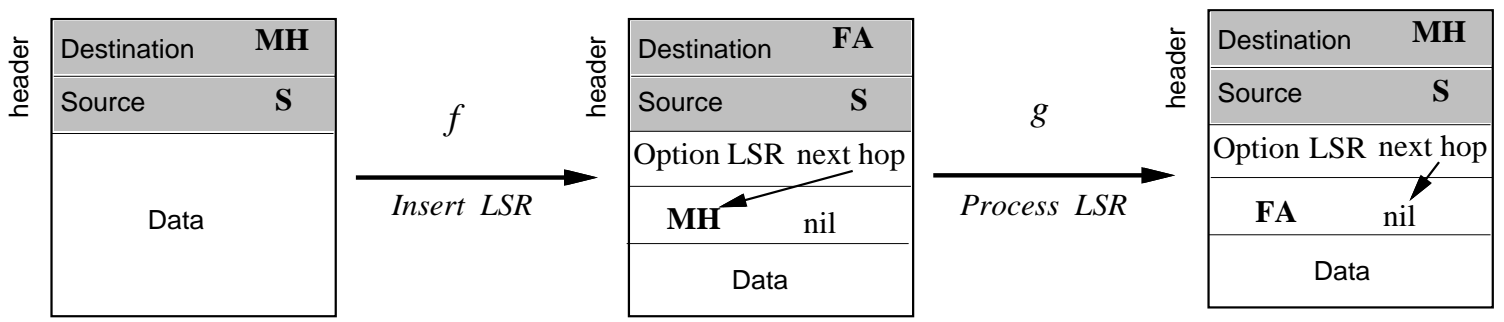

Figure 7: Using Loose Source Routing to perform address translation

An advantage of using the LSR oprion over encapsulation is that, as a natural consequence of the LSR option processing, the path that a packet follows (the list of addresses visited en-route) is automatically recorded in the packet. The destination can reverse this list and send a reply back to the source along the reverse path. In [], we show how we exploit this property to design a mobile networking scheme that co-locates the ATA with the source, and the FA with the destination. It is not possible to achieve this using any method which uses encapsulation, since when the packet arrives at the destination, it is already stripped of all useful routing information.

In this section we showed how components of the proposed architecture mutually co-operate to overlay a packet forwarding service on top of an existing routing infrastructure. It is important to point out that the ATA and the FA only represent functions that need to be supported, not machines that need to be deployed in the network. In fact, the proposed architecture allows sufficient flexibility in placement of these functions in the network. This flexibility allows us to experiment with various design alternatives and fine tune a solution for a specific target environment.

\section{Mapping to candidate MobileIP proposals}

Over the past several years, many proposals have been made for supporting host mobility on datagram-based internetworks. A vast majority of these proposals have been designed to be compatible with today's TCP/IP-based Internet. The candidate proposals differ widely in terms of the specific components they propose to add to the Internet, the mechanisms they use for address translation, and the policy they use for managing location updates. In this section, we'll show that all mobileIP proposals can be viewed as a special case of our proposed network architecture.

In our model, the ATA and FA represent the two basic functions that must be supported by any proposal that supports mobility. We'll demonstrate this fact by explaining the operation of each MobileIP proposal in terms these two functional entities. Basically, all proposals attempt 
to provide an address translation service through deployment of some additional entities in the network. They only differ in terms of their choice of where they locate these functions, the specific location update protocol they use, and whether they use encapsulation or source routing to effect address translation. Below we present a short summary of related MobileIP proposals, with a short note following each proposal outlining how its operation can be captured by our proposed solution architecture.

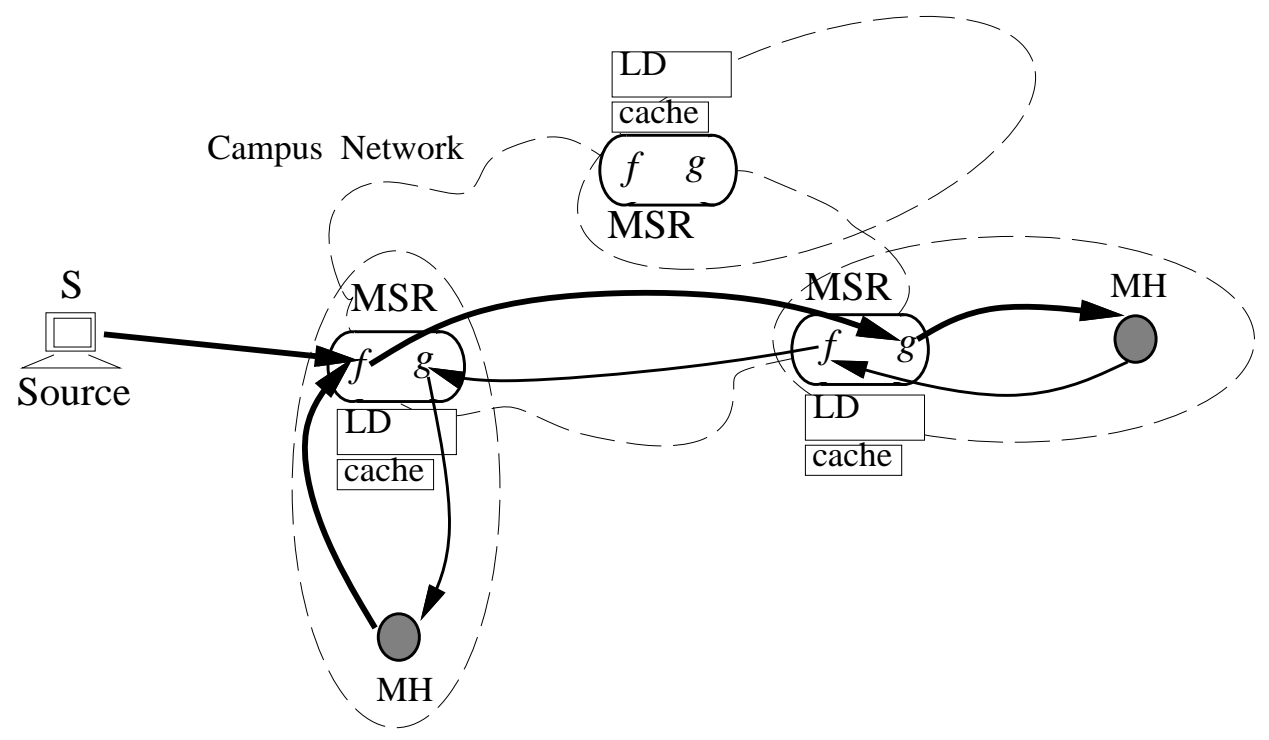

Figure 8: Mapping to Columbia Proposal

\subsection{Columbia Scheme}

The scheme proposed by Ioannidis $[7,8]$ is designed primarily to support mobility within a campus environment. Mobile hosts are allocated addresses from a subnetwork which is reserved for use by wireless hosts. A group of cooperating Mobile Support Routers (MSR), advertise reachability to the wireless subnet. MSRs provide an access point through which mobile hosts can connect to the campus back-bone, and are also responsible for forwarding traffic to and from mobile hosts. Each mobile host, regardless of its location within a campus, is always reachable via one of the MSRs. When a host sends a packet to a mobile host, it first gets delivered to the MSR closest to the source host. This MSR either delivers the packet (if the destination MH lies in its wireless cell), or forwards it to the MSR responsible for the destination MH. If an MSR does not know which MSR is currently responsible for a destination, it sends a WHO_HAS query to all MSRs in the campus and awaits a reply message from the responsible MSR. When sending a packet to the destination, an MSR encapsulates the packet and delivers it to the target MSR. Upon receiving this packet, the target MSR strips the encapsulation header and relays the original packet to the mobile host.

Mapping In the Colombia proposal, an MSR performs both encapsulation and decapsulation operations, meaning that both functions, $f$ and $g$, are co-located at the MSR. For packets addressed 
to MHs in its coverage area, an MSR acts like an FA. For packets addressed to other MHs it acts like an ATA. Each MSR maintains a table of MHs in its wireless cell. These tables together constitute the segment of the LD which is associated with mobile hosts on the campus network. This LD distribution scheme can also be thought of as a distributed realization of the owner-maintains-rule. Recall that in the owner-maintains-rule, the segment of the LD was co-located with the home router. An MSR in the Colombia scheme is a distributed realization of the home router. As a result, the table of mobile hosts maintained at an MSR constitutes a distributed segment of the $\mathrm{LD}$ that is required to be maintained at the home router.

MSRs acquire LD cache entries on a need-to-know-basis by sending a broadcast WHO_HAS query to all MSRs in the campus. The response to this query is generated by the MSR which possesses the primary copy (in other words, the MSR which is responsible for the destination $\mathrm{MH}$ ). The Location Update Protocol uses a lazy-update approach. When a mobile host moves, only the primary copy of the LD entry is updated. Cached entries are assumed to be correct by default. In cases, when cached entries turn stale, the first packet which is forwarded using the stale entry generates an error message from the old MSR, causing the source MSR to flush its cache and then broadcast a WHO_HAS message.

Since functions $f$ and $g$ are required to be supported only in new entities (MSRs) that are added to the system, the Columbia proposal can operate without requiring any modifications to the existing host and router software. This proposal presents a good combinations of design choices for handling mobility within a campus environment. However, it has severe scalability problems. Since this proposal will require broadcast of WHO_HAS query to all MSRs located world-wide, it is not possible to scale this scheme to the Internet scale.

\subsection{Sony Scheme}

In Sony's proposal $[18,16,17]$, a mobile host is assigned a new temporary address when it is attached to a new network. The router of the home network is notified of this new address through a special control message. Packets addressed to the MH, in addition to carrying its home address, can also carry its temporary address. Packets originating from an $\mathrm{MH}$ that is away from its home network always carry both home and temporary addresses in the source address field. Routers that forward these packets can examine the source addresses and cache the mapping (home to temporary) in their Address Mapping Tables (AMT). A source includes both addresses in all outgoing packets if it already has an AMT entry for the target host. Otherwise, packets are forwarded to the home address. If a transit router has an AMT cache entry for the destination, it can intercept the packet and forward it to its correct location. If none of the transit routers have a cache entry, the home router is eventually responsible for forwarding the datagram.

When a host moves to a new location, all AMT cache entries are invalidated through a special disconnect control message which is broadcast in the network. Since this message of invalidation is not reliable, there is also a timeout associated with all AMT cache entries, which, on expiration, causes AMT entries to be purged.

This method requires modifications to routers and host software and has problems interoperating with the existing hosts since it also requires modifications to IP packet formats.

Mapping: The Sony proposal co-locates the forwarding agent function, $g$, with mobile hosts. In other words, it requires each mobile host to act as its own forwarding agent. The advantage is that 


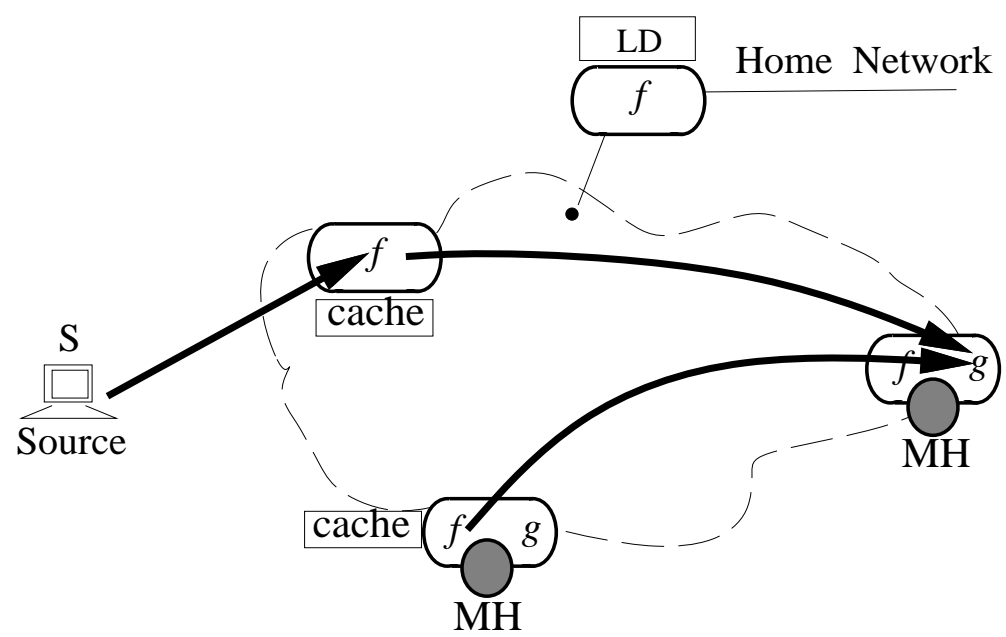

Figure 9: Mapping to Sony Proposal

packets can be directly tunneled to the mobile host, without intervention from a forwarding agent. This is useful, particularly for wired mobile hosts, which may at times connect to foreign networks which have no forwarding agents attached. The approach of co-locating $g$ with the mobile host has a disadvantage. It doubles the address space requirement for mobile hosts, since in addition to a home address, a temporary address is also required for operation. Given that IP address space is fast running out of available addresses, this is a serious problem.

In Sony's proposal, the home router acts as an address translation agent $(f)$, and it also maintains the Location Directory for mobile hosts that have been assigned addresses on the home network. To avoid routing each packet via the home router, Sony proposal allows flexibility to co-locate $f$ with internet routers. Since LD cache entries are carried in the source address field of the VIP protocol ${ }^{5}$, routers can acquire these them just by inspecting the source address of packets they relay. Distributing LD caches all over the Internet improves routing performance; however, it makes updates very costly. Sony's proposal, therefore, has severe scalability problem. When a host moves to a new location, it is required to send a broadcast in the network to purge all cached LD entries.

\subsection{MobileIP working-group Proposal}

IETF has created a MobileIP working group to come up with a proposal for near term deployment within the Internet. In this design [11], each mobile host retains its home address regardless of the mobile host's location. When the mobile host visits a foreign network, it is associated with a care-of-address, which is an Internet address associated with the mobile host's current point of attachment. The care-of-address either identifies the mobile host directly (if the address is acquired through Dynamic Host Configuration Protocol (DHCP)) or identifies a Foreign Agent that is responsible for providing access to visiting mobile hosts. When away from home, the mobile host registers its care-of-address with a Home Agent; the Home Agent is responsible for intercepting datagrams addressed to the mobile host's home address and tunneling (encapsulating) them to the

\footnotetext{
${ }^{5}$ The modified IP protocol
} 


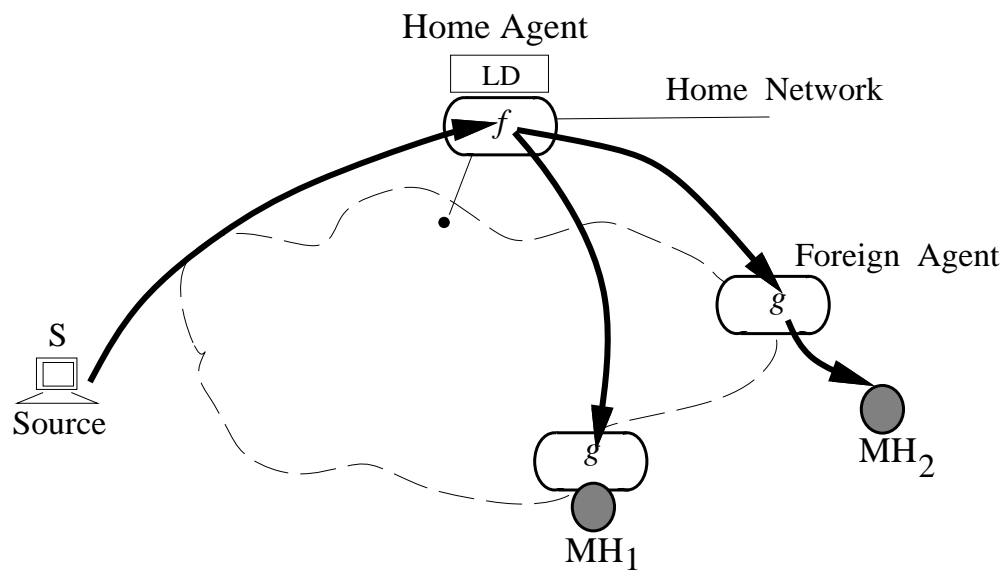

Mobile Host using DHCP

Figure 10: Triangle Routing: MobileIP Proposal

associated care-of-address.

In this scheme all datagrams addressed to a mobile host are always routed via the Home Agent. However, the packets in the reverse direction, i.e., those originating from the mobile host and addressed to a stationary host, are relayed along the shortest path by the Internet routing system. This gives rise to what is known as the triangle routing problem. Route optimization is possible if the location information is allowed to be cached; however, this proposal does not permit caching of LD entries because of security concerns. Currently, the Internet does not provided any secure mechanism for distributing cache entries. Any entity in the Internet can masquerade as a Home Agent and re-route traffic away from a mobile host just by re-distributing fake cache entries. This proposal, therefore, takes the stand that routing based on cached location information is insecure, and the best possible defense against security attacks is to not use it at all. The cost of this choice is that routing is always non-optimal.

When the mobile host arrives at a foreign network, it can listen for (or solicit) agent advertisements to determine whether a Foreign Agent is available. If so, the registration request to the Home Agent is sent via the Foreign Agent; otherwise, the mobile host must acquire a care-of-address (through DHCP), and then register with the Home Agent.

Mapping: The IETF-MobileIP proposal reflects a design choice that co-locates $f$ with the Home Agent and $g$ with the Foreign Agent. This proposal also allows $g$ to be co-located with the mobile host. This happens when the mobile host acquires a temporary address via DHCP. The location update protocol is very simple; the mobile host notifies the Home Agent whenever it moves to a new location. Since the LD entries are never cached, the question of maintaining consistency doesn't even arise.

\subsection{LSR Scheme}

In contrast with other proposals which are encapsulation based, the LSR proposal $[5,12,13,9]$ is based on the use of an existing IP option called Loose Source Route. The LSR scheme also allows each mobile host to retain its home address regardless of its current location. Associated with each 


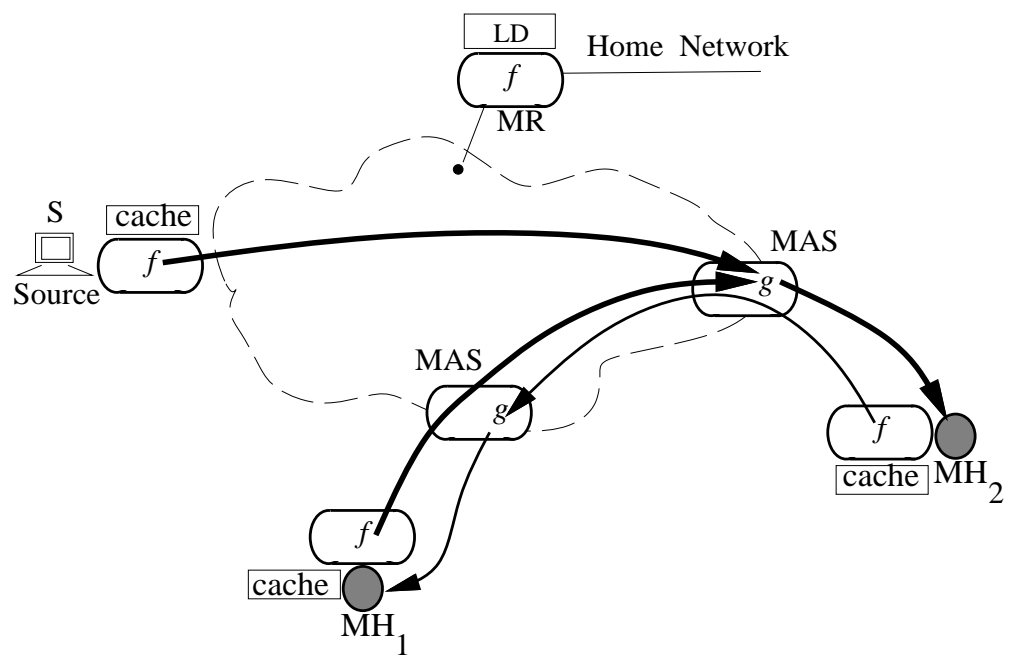

Figure 11: Mapping to LSR Scheme

home network is a Mobile Router, which is responsible for advertising reachability to the home network, and for keeping track of the current location of each mobile host that has been assigned an address on that network. In a foreign network, mobile hosts attach to the Internet via wireless base stations known as Mobile Access Stations (MAS). When a mobile host walks into the wireless cell of an MAS, it informs its Mobile Router the internet address of the current MAS. The Mobile Router records this information in its routing table, and also informs the previously recorded MAS that the mobile host has migrated from its wireless cell. The packets sent to the mobile host first arrive at the Mobile Router by the normal routing process. To forward a packet to the a mobile host's current location, the Mobile Router inserts an LSR option in the packet, specifying the current MAS as a transit router. The inserted LSR option causes this packet to be routed to the mobile host via the MAS. When the mobile host sends a reply to the source, it also inserts the LSR option in all outgoing packets, again specifying the current MAS as a transit router. When the stationary host receives this packet, it will reverse the recorded route, and insert it in all outgoing packets that are sent to the mobile host. Thus, subsequent packets originating from the stationary host will be automatically routed along an optimal path. Notice that route reversal is an integral part of LSR option processing. The LSR scheme exploits this feature to provide optimal routing between stationary and mobile hosts.

Mapping: In this proposal, the MR acts as an ATA, and is also responsible for maintaining the LD. The MAS acts an FA for mobile hosts that lie in its wireless cell. The key feature of this proposal is that it enables function $f$ to be co-located with all internet hosts without requiring changes to host software. All internet hosts, when generating replies to packets that are received with the LSR option, are required to do the route reversal[6]. For TCP connections, the route reversal is performed by the protocol processing module, and in case of UDP connections, this responsibility lies with the applications. From our reference architecture view point, the process of route reversal amounts to the task that an ATA is required to carry out. Thus, this scheme effectively exploits mechanisms already available within IP protocol, and achieves co-location of 
ATA with end hosts without requiring any modifications to host software. It is worth mentioning that this feature cannot be achieved using any scheme that is based on encapsulation. Unlike LSR, encapsulation is not a part of the standard IP protocol specification. Therefore, no internet host can generate encapsulated IP packets without suitable software modification.

Another important feature of this scheme is that no special protocol is required for distributing and managing LD cache entries. LD entries are automatically acquired through the incoming LSR option. Recall that packets which arrive at a stationary host already contain the address of the MAS. This, together with the source address of the packet, constitutes an LD cache entry. When a host starts a new session with a mobile host, it has no LD cache entry for the destination. Naturally, the first packet is routed to the destination via the MR. When the ACK for this packet arrives, it contains the LD cache entry ${ }^{6}$ in the incoming LSR option. This LD entry is maintained on a per-session basis, and it maintained only as long as the corresponding TCP session is alive. When the session terminates, the corresponding LD entry is purged. If the destination moves during an active session, the LD cache entry becomes inconsistent. However, it gets updated as soon as the next packet from the destination arrives at the source. This constitutes a pure on-demandcache-update policy which has a good scaling property. Following a host's movement, only those LD cache entries are updated which are in use. Compared with Sony's proposal, which requires a message to be broadcast to the network, significantly fewer messages are exchanged. Naturally, an on-demand-cache-update policy lends a scalable design; both with respect to the size of the network, and the rate of host mobility.

\section{Summary}

In this paper, we first identified network layer concepts that play a crucial role in the design of mobile networking systems. We showed that the process of address translation is fundamental to providing any solution to mobility at the network layer. Our proposed network architecture employs three basic set of entities: Address Translation Agent, Forwarding Agent, and Location Directory, which co-operate with each other to carry out the operation of address translation. The proposed architecture is general and flexible. The architecture's generality enables it to capture all possible scenarios of communication between mobile and stationary hosts. Its flexibility allows sufficient freedom in terms of placement of these entities in the network.

We showed that all candidate proposals for MobileIP can be visualized as special cases of our proposed architecture. We demonstrated this by showing a one-to-one mapping between the entities in our architecture, and those required by the candidate proposals. Mappings represent set of design choices (i.e., where in the network these entities are located) made in the candidate proposals (see Table 2).

In addition to these design choices, there are several other considerations such as inter-operability, backward-compatibility, security, and authentication, which also play a crucial role in the design of a mobile networking system. Interested readers can refer to articles $[17,19,8,10]$ for an in-depth description of design and implementation issues.

\footnotetext{
${ }^{6}$ All BSD 4.3 compliant TCP implementations copy this information in the TCP control block
} 


\section{References}

[1] V. Anantharam, M. L. Honing, U Madhow, and V. K. Wei. Optimization of a database hierarchy for mobility tracking in a personal communications network. In Proceedings of Performance 93, Septembero 1993.

[2] Baruch Awerbuch and David Peled. Online Tracking on mobile users. In Proceedings of ACM SIGCOMM, 1991.

[3] Amotz Bar-Noy and Ilan Kessler. Tracking Mobile Users in wireless communication Networks. IEEE Transactions on Information Theory, pages 45-65, Jan 1994.

[4] Amotz Bar-Noy, Tlan Kessler, and Moshe Sidi. Mobile Users: To Update or not to Update? In Proceedings of IEEE INFOCOM, pages 570-576, Toronto, Canada, June 1994.

[5] Pravin Bhagwat and Charles Perkins. A Mobile Networking System based on Internet Protocol(IP). In Proceedings of USENIX Symposium on Mobile and Location Independent Computing, pages 69-82, Cambridge, MA, Aug 1993.

[6] R. Braden. Requirements for Internet Hosts - Communication Layers. RFC 1122, Oct. 1989.

[7] John Ioannidis, Dan Duchamp, and Gerald Q. Maguire Jr. IP-based Protocols for Mobile Internetworking. In Proceedings of ACM SIGCOMM, pages 235-245, 1991.

[8] John Ioannidis and Gerald Q. Maguire Jr. The Design and Implementation of a Mobile Internetworking Architecture. In Proceedings of Winter USENIX, pages 491-502, San Diego, CA, Jan 1993.

[9] David B. Johnson. Mobile host internetworking using ip loose source routing. Technical Report CMU-CS-93-128, Carnegie Mellon University, Pittsburgh, PA 15213, February 1993.

[10] Andrew Myles and David Skellern. Comparing Four IP Based Mobile Host Protocols. In Joint European Networking Conference, May 1993.

[11] Charles Perkins. draft-ietf-mobileip-protocol-09.txt. Draft RFC - work in progress, June 1995.

[12] Charles Perkins and Pravin Bhagwat. A Mobile Networking System based on Internet Protocol. IEEE Personal Communication Magazine, 1(1):32-41, Feb 1994.

[13] Charles Perkins and Yakov Rekhter. Short-cut Routing for Mobile Hosts. Internet draft, July 1992.

[14] J. Postel. Internet Protocol. RFC 791, Sep 1981.

[15] J. Postel. Transmission Control Protocol. RFC 793, Sep 1981.

[16] Fumio Teraoka and Mario Tokoro. Host Migration Transparency in IP Networks. Computer Communication Review, pages 45-65, Jan 1993.

[17] Fumio Teraoka, Keisuke Uehara, Hideki Sunahara, and Jun Murai. VIP: A Protocol Providing Host Mobility. Comminications of the ACM, 37(8), August 1994. 
[18] Fumio Teraoka, Yasuhiko Yokote, and Mario Tokoro. A Network Architecture Providing Host Migration Transparency. In Proceeding of ACM SIGCOMM, Sept 1991.

[19] Hiromi Wada, Takashi Yozawa, Tatsuya Ohnishi, and Yasunori Tanaka. Mobile Computing Environment Based on Internet Packet Forwarding. In proceeding of Winter USENIX, pages 503-517, San Diego, CA, Jan 1993. 


\begin{tabular}{|l|l|l|l|l|}
\hline \hline Scheme & $\begin{array}{l}\text { Address Translation } \\
\text { Agent }(f)\end{array}$ & Forwarding Agent $(g)$ & Location Directory & Location Update Protocol \\
\hline \hline Columbia & $\begin{array}{l}\text { Co-located with } \\
\text { MSR }\end{array}$ & Co-located with MSR & distributed among MSRs & $\begin{array}{l}\text { Only primary copy is modified. } \\
\text { Lazy-update policy is used for up- } \\
\text { dating cache entries }\end{array}$ \\
\hline Sony & $\begin{array}{l}\text { Co-located with all } \\
\text { hosts and routers }\end{array}$ & $\begin{array}{l}\text { Co-located with mobile } \\
\text { hosts }\end{array}$ & $\begin{array}{l}\text { LD is maintained at home } \\
\text { Only primary copy is modified } \\
\text { quired by snooping a packet } \\
\text { hy the explicit connect message. } \\
\text { Cache entries are modified by } \\
\text { broadcasting a disconnect mes- } \\
\text { sage, or are auto-flushed by a } \\
\text { timeout mechanism }\end{array}$ \\
\hline $\begin{array}{l}\text { MobileIP } \\
\text { working group }\end{array}$ & $\begin{array}{l}\text { Co-located with } \\
\text { home routers }\end{array}$ & $\begin{array}{l}\text { Co-located with Foreign } \\
\text { Agent, or with mobile } \\
\text { host if DHCP is used. }\end{array}$ & $\begin{array}{l}\text { LD is maintained at home } \\
\text { router only. }\end{array}$ & $\begin{array}{l}\text { Due to security reasons, caching } \\
\text { of LD entries is not allowed. This } \\
\text { implies when a host moves only } \\
\text { the primary copy is required to } \\
\text { be modified. A simple location } \\
\text { update message from the mobile } \\
\text { host suffices for this purpose. }\end{array}$ \\
\hline LSR scheme & $\begin{array}{l}\text { Co-located with } \\
\text { all hosts and home } \\
\text { routers }\end{array}$ & $\begin{array}{l}\text { Co-located with mobile } \\
\text { hosts }\end{array}$ & $\begin{array}{l}\text { LD is maintained at home } \\
\text { router. Cache entries are ac- } \\
\text { quired through incoming LSR } \\
\text { option }\end{array}$ & $\begin{array}{l}\text { Only primary copy is modified. } \\
\text { Capdated when automatically get } \\
\text { uSR option arrive. On-demand } \\
\text { update policy, no broadcasts. }\end{array}$ \\
\hline
\end{tabular}




\begin{tabular}{|c|c|c|c|c|c|}
\hline \multirow{2}{*}{ Property } & \multirow[t]{2}{*}{ 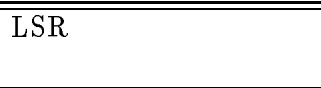 } & \multicolumn{2}{|c|}{ Columbia } & \multirow[t]{2}{*}{$\overline{\text { Sony }}$} & \multirow{2}{*}{ IETF Mobile-IP } \\
\hline & & In-campus & Out-of-campus & & \\
\hline Optimal Routing & Always & Always & Never & $\begin{array}{l}\text { Only if all routers are } \\
\text { modified }\end{array}$ & Never \\
\hline $\begin{array}{l}\text { Address Translation } \\
\text { Mechanism }\end{array}$ & Loose Source Routing & \multicolumn{2}{|c|}{ Encapsulation } & Encapsulation & Encapsulation \\
\hline $\begin{array}{l}\text { Additional Address } \\
\text { Space Required }\end{array}$ & None & None & Double & Double & $\begin{array}{l}\text { None. But required when us- } \\
\text { ing DHCP. }\end{array}$ \\
\hline Failure Modes & $\begin{array}{l}\text { MR is a single point of } \\
\text { failure, but it does not } \\
\text { affect on-going sessions }\end{array}$ & $\begin{array}{l}\text { Robust } a \\
\text { MSR failur }\end{array}$ & $\begin{array}{ll}\text { ainst local } \\
\text { s }\end{array}$ & Non-local & $\begin{array}{l}\text { Home Agent is a single point } \\
\text { of failure, and it affects all on- } \\
\text { going sessions }\end{array}$ \\
\hline Scalability & Good & & Good & Poor & \begin{tabular}{|l|} 
Excellent \\
\end{tabular} \\
\hline Compatibility with IP & $\begin{array}{l}\text { So long as hosts } \\
\text { and routers conform to } \\
\text { standards }\end{array}$ & & Total & Requires changes & Total \\
\hline Security & Insecure & Parti & 11 lly Secure & Insecure & Fully Secure \\
\hline
\end{tabular}

Table 1: Property Comparison of MobileIP schemes 